\title{
Dental Education in Europe
}

Modern dental education has its roots in the USA where the first dental school started in Baltimore in 1840. It did not take long, however, before independent dental curricula were introduced in Europe leading to different patterns of education. During the last thirty years different activities in harmonization of European higher education have lead dental education also to face continuous changes.

The European Community decided to harmonize different professional degrees by developing directives for health sector. The dental directive defining an independent dental degree based on five year curriculum was issued in 1978. The Advisory Committee for the Training of Dental Practitioners was established to advise the EC on further curricular development. Regardless of beneficial work by the Advisory Committee on development of European dental curricula its function came to an end in 2000. Also the dental directive was merged to a new one on the recognition of professional qualifications in 2005. This Directive includes the educational requirements concerning the mutual recognition of Diplomas, Certificates and other evidence of the formal qualifications of dental practitioners.

The Association for Dental Education in Europe (www.adee.org) has served as the forum of discussion of models and experiences in dental education since its foundation in 1975. During its existence the Association has grown into a professional organization having more than 160 dental schools as members and running a central office in Dublin. ADEE represents the voice of dental education in Europe, the European Union and the European Higher Education Area.

Over the years ADEE has also been active in pursuing for high quality in dental education. Under its patronage, the Thematic Network Project DentEd was first supported by the EU in 1997 to facilitate convergence towards higher standards in dental education and professional training. This project has been able to establish a wide network of dental schools by creating a model for peer evaluation, based on selfassessment and peer-review visits and has identified innovative models in attaining high standards in dental education throughout Europe. After the first phase of DentEd, a new project DentEdEvolves was funded by the EU during 2000-2003. This project continued the visitation programs and aimed to widen the harmonization process beyond European borders. ADEE together with DentEdEvolves network has obtained further funding from the EU for the newest project DentEd III for 20042007. During DentEd III it has been possible to develop a curricular model for dentistry compliant with the principles of the Bologna Declaration.

In 1999 the 29 Ministries of Education of the European Union countries signed the socalled Bologna Declaration placing special emphasis on the need to provide the European area of higher education with more coherence while maintaining the inherent diversity. Today the process unites 46 countries to converge and harmonize the higher educational systems in Europe. One of the objectives is to tune curricula in terms of structures, programmes and actual teaching in order to make them more comparable. Thus it is deemed to facilitate mobility of both staff and students; a single European economic area goes hand in hand with a single European higher education area.

The Association of Dental Education in Europe has been very active to support dental educators to introduce necessary changes to allow real development in European dental education. ADEE in collaboration with DentEd has elaborated a document on Profile and Competences of The European Dentist. This document, easily accessible on ADEE web site, contains seven domains with a minimal set of major and supporting competences. It was approved by the General Assembly of ADEE 
in 2004 and has been translated into several European languages. The ultimate goal of this document is that all European dental schools will adhere to the major competences to further improve the quality of their education. To meet the needs caused by continuous changes in our societies the document is presently under review process to be presented to the General Assembly in ADEE Helsinki Annual Meeting in August 2009 (www.hammas.helsinki.fi/ADEE2009).

According to ADEE the dental curriculum in addition to necessity of reflecting history, culture and political system of a country should also contribute to the oral health needs of local population and it should be organized in a modular form. These modules should aim to integrate knowledge around problems, conditions or preventive and therapeutic goals. The present explosion of information requires an approach from teachers and students that moves away from merely learning facts to a more problem-solving approach and appropriate use of information technology. All dental schools should also try to remove obstacles to mobility. Mobility of students and academic/ administrative staff is the basis for establishing a joint European Higher Education Area.

Curriculum Structure and Benchmarking \& Quality Assurance are other documents produced by ADEE to facilitate harmonization. In European Higher Education the Declaration of Bologna advocates the modularisation of programs with mutual recognition across national boundaries whilst implementing a European Credit Transfer and Accumulation System (ECTS) in order to promote exchange. The ADEE Curriculum Structure document provides the suggested framework for a dental educational model, which will serve as a template for use within various national systems. The framework includes aspects of learning outcomes, content, structure, student exchange and ECTS. The Bologna signatories have also agreed standards and guidelines for quality assurance within the European Higher Education Area. The ADEE Benchmarking and Quality Assurance document provides a guide to assist in the harmonization of dental education quality assurance systems across Europe.

The DentEd project has proved its merits and benefits by promoting convergence towards higher standard of dental education, training and service to the ultimate benefit of the patients. The final phase of the DentEd culminated in the Global Congress in Dublin in last September where all continents will be represented. Already in 1992 ADEE had widened its horizons by becoming a founding member of the International Federation of Dental Education Associations. IFDEA links all the regional dental education organisations into a single federation. ADEE is proud and pleased to continue collaboration with all continental dental education associations and a global community of dental educators to improve oral health worldwide by sharing knowledge and improving standards through the Global Network on Dental Education (www.ifdea.org).

All of us should be happy to witness that ADEE has been on the front seat navigating European dental education on the way towards the goals set for Higher Education Area according to the action lines of Bologna Declaration. For the first time in the history of European dental education core documents have been approved to lay welljustified and harmonized basis for training high quality dentists to better serve those in need. This is a good beginning and everybody in the dental profession should now be proud of the future dentists. However, we should not forget that the developments in educational sciences let alone dentistry in general are presently very fast. The constant actions in up-dating of knowledge are of paramount importance and a responsibility of each oral health care professional. In this respect we all are, from a first year student to most experienced practitioner, in the same vessel! Fortunately these activities can be today carried also at the European level and not only locally or nationally. 